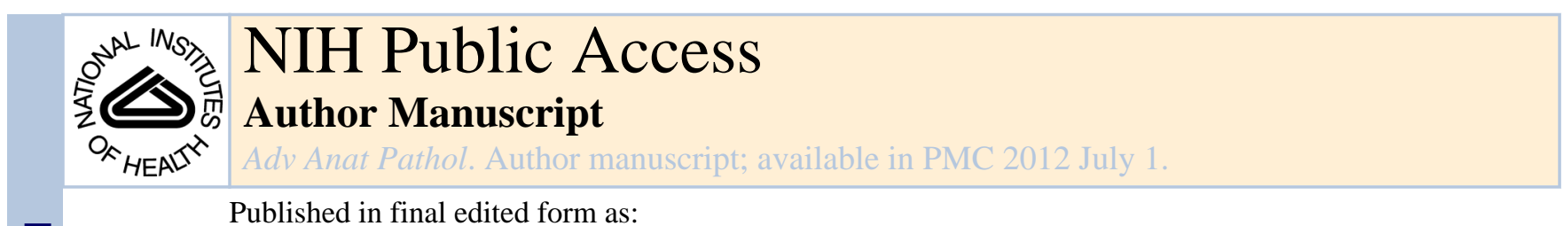

Published in final edited form as:

Adv Anat Pathol. 2011 July ; 18(4): 294-300. doi:10.1097/PAP.0b013e318220f59b.

\title{
UPDATE ON FATTY LIVER DISEASE AND STEATOHEPATITIS
}

\author{
F. Zahra Aly, M.D, Ph.D and David Kleiner, M.D, Ph.D* \\ Laboratory of Pathology, National Cancer Institute, National Institutes of Health, Bethesda, MD \\ 20892
}

\section{Keywords}

NASH; NAFLD; steatosis; steatohepatitis; adults; pediatric; pathology

\section{Introduction}

Numerous publications have highlighted a global epidemic of obesity which not only affects the Western world but is also evident in rapidly developing countries [1-2]. Obesity used to be a disease mainly of adults; however, it is now recognized to be a major problem in children as well. Concerns have been raised about the rising incidence of obesity coupled with increasing awareness of its major co-morbidities: cardiovascular events and diabetes [4]. Obesity in children is also associated with poor health outcomes, including early onset of diabetes and hypertension.

The major manifestation of obesity in the liver is non-alcoholic fatty liver disease (NAFLD) [5]. This may be clinically silent or present with altered liver enzymes, vague upper right quadrant abdominal symptoms or significant malaise. Although a plethora of non-invasive tests for diagnosing NAFLD have emerged in recent years, the gold standard for assessing all aspects of this complex disease remains the liver biopsy. Liver biopsy cannot be used as a screening tool and so general population prevalence studies have used imaging and/or serological tests to assess NAFLD.

Histopathologically, NAFLD encompasses a spectrum ranging from isolated steatosis, steatohepatitis and progressive forms to cirrhosis. Clinically significant steatosis is defined as abnormal lipid deposition in hepatocytes ( $>5 \%$ hepatocytes or liver weight) in the absence of excess alcohol intake. Recent studies suggest that hepatic steatosis is present in more than $60 \%$ of obese and $90 \%$ of morbidly obese adults [6-7].

Non alcoholic steatohepatitis (NASH) is defined as a particular pattern of injury with steatosis, hepatocellular ballooning and inflammation. About $2-3 \%$ of the general population is estimated to have NASH [9]. Among patients with metabolic syndrome (Text box 1), the prevalence of NAFLD was 74\%. The progression of steatosis to NASH is estimated to be $19 \%$ in obese and $50 \%$ in morbidly obese adults [7]. Subsequent progression to liver fibrosis or cirrhosis occurs in approximately $40 \%$ [11].

*Corresponding author: Dr D E Kleiner, Laboratory of Pathology, National Cancer Institute, National Institutes of Health, Bethesda, MD 20892. Telephone: 301-496 2441, Fax: 301-480 9488, kleinerd@ mail.nih.gov.

Publisher's Disclaimer: This is a PDF file of an unedited manuscript that has been accepted for publication. As a service to our customers we are providing this early version of the manuscript. The manuscript will undergo copyediting, typesetting, and review of the resulting proof before it is published in its final citable form. Please note that during the production process errors may be discovered which could affect the content, and all legal disclaimers that apply to the journal pertain. 
The prevalence of pediatric NAFLD is unknown. Indirect data such as the National Health And Nutrition Examination Survey (NHANES) study suggest that $8 \%$ of US adolescents (12-19 y) have unexplained elevated alanine aminotransferase [12]. A population based autopsy series of 742 children in San Diego demonstrated a prevalence of NAFLD to be $9.6 \%$ overall, with up to $17 \%$ among adolescents [14]. The prevalence of the disease is increased among obese subjects. NAFLD is reported to affect $20 \%$ of obese children and adolescents from the United States [15]. In the largest two studies of biopsy proven NAFLD described in the literature [16-17], NASH was diagnosed in $84 \%$ and $68 \%$, respectively.

The increased prevalence of obesity as well as the availability of therapies directed against NASH [18] increases the likelihood that pathologists will need to evaluate liver biopsies that demonstrate the morphologic changes of NAFLD. The scope of the present article is to 1) review the histological features of steatosis and non-alcoholic steatohepatitis in adults 2) review the variation of histologic patterns of pediatric fatty liver disease, and, 3) discuss the validity and utility of the NAFLD Activity Score (NAS).

\section{Text Box 1}

At least 3 organizations have recommended clinical criteria for the diagnosis of the metabolic syndrome. These are the National Cholesterol Education Program (NCEP)[3], World Health Organization (WHO)[8] and American Association of Clinical Endocrinologists (AACE) [10].

The US National Cholesterol Education Program Adult Treatment Panel III (2001) requires at least three of the following:

- central obesity: waist circumference $\geq 102 \mathrm{~cm}$ or 40 inches (male), $\geq 88 \mathrm{~cm}$ or 36 inches(female)

- dyslipidemia: $\mathrm{TG} \geq 1.7 \mathrm{mmol} / \mathrm{L}(150 \mathrm{mg} / \mathrm{dl})$

- dyslipidemia: HDL-C $<40 \mathrm{mg} / \mathrm{dL}$ (male), $<50 \mathrm{mg} / \mathrm{dL}$ (female)

- blood pressure $\geq 130 / 85 \mathrm{mmHg}$

A recent proposal by the International Diabetes Federation Group[13] broadly confirms the premise of the NCEP definition but introduces ethnicity based waist circumference figures and incorporates the most recent diagnostic level from the American Diabetes Association for impaired fasting glucose $(5.6 \mathrm{mmol} / \mathrm{L}$ [100 mg/dL]).

\section{Histologic Features of Steatosis in Adults}

Steatosis is a necessary component for the diagnosis of both NAFLD and NASH. It is defined as macrovesicular or microvesicular triglyceride accumulation involving at least 5\% of hepatocytes. In macrovesicular steatosis, a single large droplet displaces the cytoplasmic contents and the nucleus to the periphery of the cell. Hepatocytes with macrovesicular steatosis may also contain smaller droplets in a ring formation around the larger one or several small droplets without a larger droplet (Figure 1). True microvesicular steatosis imparts a foamy appearance to the cytoplasm from the innumerable tiny vacuoles and usually indents the centrally retained nucleus (Figure 2). In NAFLD, microvesicular steatosis may be present as single cells or patches of contiguous hepatocytes. Megamitochondria are often seen within these patches (Figure 3). A unifying hypothesis explaining the association of megamitochondria and microvesicular steatosis might be the abnormal oxidative ability of the cell reflected by megamitochondria resulting in oxidative injury of the cell in the form of microvesicular steatosis and disruption of the cytoskeleton as seen by ballooning cell changes and Mallory Denk bodies (MDB) (Figure 4)[19]. 
Microvesicular steatosis is seen in $10 \%$ of liver biopsies obtained from patients with NAFLD. This form of steatosis has been shown to correlate with more advanced histologic features of NAFLD i.e. higher grade of steatosis, ballooning cell injury and more fibrosis [19]. In contrast, the severity of macrosteatosis is related to lobular inflammation but not to other components of NASH histology like ballooning, MDB or fibrosis [20].

\section{Histological Features of Steatohepatitis in Adults}

Steatohepatitis is a histologic pattern of hepatic injury characterized by steatosis, inflammation, hepatocellular ballooning and fibrosis. The initial injury in adults is usually in the perivenular (zone3) portion of the hepatic acinus which is least oxygenated and most prone to damage by oxidizing free radicals.

The key to histologic diagnosis is identification of hepatocyte injury which is characterized by ballooned hepatocytes (Figure 4). This alteration is characterized by the presence of enlarged hepatocytes with a characteristic flocculent or irregularly clumped appearance to the cytoplasm. There may or may not be residual fat droplets within these cells. In addition, MDB may or may not be present.

As ballooning is central to the distinction between steatosis and steatohepatitis, efforts to analyze it have included immunohistochemical and ultrastructural studies.

Immunohistochemical studies have shown differential expression of keratins and lipid droplet associated proteins. The two hepatocyte keratins, K8 and K18 are disrupted and no longer present throughout the cytoplasm of ballooned hepatocytes; rather they are dispersed to the periphery. This has been proposed as an objective marker for the histological recognition of ballooning cell injury [21]. Recent studies highlight the altered expression of lipid droplet associated proteins perilipin 1 and 2 (but not perilipin 3), and,

immunohistological co-localization of oxidized phosphatidylcholine in ballooned hepatocytes [22] [23]. These two new findings have not been investigated further and their diagnostic utility is therefore unknown at this time. Ultrastructural study of ballooned cells demonstrated the presence of lipid droplets in hepatocytes that were larger than 20 microns [24]. Because of the difficulty in identifying ballooned cells in ultrastructural preparations, it is unlikely that this feature is useful on routine basis.

Inflammation is present to a variable degree and is usually more prominent in the lobule than the portal area (zone 3). The inflammatory cells are most often lymphocytes, but may include neutrophils, eosinophils and/or activated Kuppfer cells. Neutrophils may accumulate around hepatocytes (satellitosis) that contain MDB. Apoptotic hepatocytes (acidophil bodies), cleared hepatocyte nuclei filled with glycogen, non-anatomically distributed 'patches' of hepatocytes with true microvesicular steatosis may also be seen. As noted above, cells with true microvesicular steatosis may harbor megamitochondria; however megamitochondria may also be seen in ballooned hepatocytes and within hepatocytes that are otherwise unremarkable.

The effector cells of the innate immune system residing in the liver react by forming clusters of Kuppfer cells (microgranulomas) (Figure 5). The increase in Kupffer cell aggregates is associated with their migratory shift from their normal position in zone 1 (periportal) to zone 3 (perivenular) [25]. Lipogranulomas are aggregates of macrophages containing lipid droplets. They may be seen in perivenular, lobular or portal areas. They are often associated with local fibrosis and lipogranuloma-associated fibrosis should not be considered as part of the fibrosis of steatohepatitis (Figure 6).

Portal chronic inflammation is generally mild in steatohepatitis in comparison with that seen in chronic hepatitis. A NASH Central Research Network (NASH CRN) biopsy study 
focused on the relationship of portal inflammation to other histologic, demographic and laboratory features [26]. There was strong correlation between increased portal inflammation and the stage of liver disease as well as the presence of ballooning cell changes. There was no association between portal fibrosis and degree of lobular inflammation, ALT or autoimmune markers (ANA and ASMA). Clinically, portal inflammation was mainly associated with parameters of insulin resistance [obesity, homeostatic model assessment of insulin resistance (HOMA-IR), insulin levels and medications used for diabetes]. While the mechanism invoked for the presence of lobular inflammation is hypoxic oxidative injury, the mechanism underlying the presence of portal tract chronic inflammation is not known. Of note is that increased portal inflammation in adults may also follow therapy with glitazones. When moderate or marked inflammation is seen, it is prudent to consider the presence of other concurrent diseases such as chronic hepatitis, autoimmune hepatitis and chronic cholestatic disease before attributing the portal inflammation solely to steatohepatitis. Large biopsy series have histologic evidence of concurrent steatohepatitis in up to 5\% of patients with a serologically diagnosed liver disease such as chronic hepatitis $\mathrm{C}$, autoimmune liver disease or primary biliary cirrhosis [27-28]. Infection with hepatitis C virus genotype 3 is characterized by hepatic steatosis and may be confused with NASH unless the specific features of NASH are seen.

The pattern of fibrosis in steatohepatitis is distinctive and can be very helpful in making the diagnosis of steatohepatitis. The fibrosis is perisinusoidal in distribution and usually begins or is more prominent in zone 3. It may be delicate and visualized only with the aid of special stains, or may be dense and evident on routine stains (Figure 7). With progression, linkage of vascular structures results in bridging fibrosis and remodeling of the hepatic parenchyma which finally results in cirrhosis. There is general agreement that progression of fibrosis in adult NASH begins with perisinusoidal fibrosis followed by other patterns. Portal fibrosis may develop with progression and is often seen in the presence of pericellular fibrosis. With development of bridging fibrosis and cirrhosis, pericellular fibrosis may be lost. A recent study has shown that elastic fibers (connoting maturity of collagen deposits) are present in the bridging and cirrhotic phases and may be useful in deriving stage in questionable cases [29]. Of interest is that dense perisinusoidal fibrosis referred to as hepatosclerosis may occur in Type 1 diabetics. This pattern is not associated with steatosis or necroinflammatory activity [30].

The factors involved in hepatic fibrogenesis are being elucidated. A major focus has been on the reaction of the lobular stellate cells and hepatic progenitor cells to chronic inflammatory stimulus. Activation of the stellate cells leads to pericellular fibrosis. The hepatic progenitor cells are activated and proliferate in the setting of chronic hepatocyte injury and are associated with ductular reaction and periportal fibrosis. There is evidence of epithelial to mesenchymal transition of ductular cells via activation of the hedgehog pathway, a process by which mature epithelial cells differentiate into cells with a mesenchymal phenotype [31]. The degree of ductular reaction is correlated with the grade of NASH activity, the degree of fibrosis, and the extent of primary hepatocyte replicative arrest, which are in turn correlated with insulin resistance [32]. In multivariate analysis, fibrosis was associated independently with ductular reaction, hepatocyte ballooning and portal inflammation.

Increased hepatic iron may also have a role in the development of NASH. Insulin resistance is associated with increased hepatic iron levels [33]; improved glycemic control is associated with improvements in serum ferritin and hepatic iron concentrations [34]; increased parenchymal hepatic iron concentration in NASH appears to correlate with the severity of fibrosis [35]. The specific mechanism by which hepatic iron may contribute to necroinflammation is unknown, but may be related to the generation of free oxygen radical species that occurs in the process of reduction of Fe3+to Fe2+ [36]. Still unexplained is the 
observation that homozygosity for hemochromatosis gene mutation does not appear to confer an increased risk for the development of NAFLD [37]. Furthermore, the clinical significance of iron overload in NASH with respect to clinically relevant endpoints is unclear. In an unselected cohort of 65 patients with NASH, iron accumulation was not associated with increased overall mortality, liver related mortality or development of cirrhosis [38]. A recent publication showed that patients with the reticuloendothelial system (RES) iron-staining pattern were more likely to have advanced fibrosis compared to those with hepatocellular iron. Patients with RES iron were also more likely to have advanced histological features such as fibrosis, portal inflammation, ballooning, and definite steatohepatitis compared to patients with hepatocellular or mixed iron patterns. The presence of RES iron was independently associated with advanced hepatic fibrosis on multiple regression analysis after adjustments for age, gender, diabetes status, and body mass index [39].

In summary, the key pathologic features of steatohepatitis in adults are zone 3 centered hepatocellular injury with ballooning cell changes. Steatosis and inflammation are present but variable in degree. MDB and pericellular fibrosis are very useful in diagnosis; however, they are not required if zone 3 injury (ballooning changes) is definitely demonstrated.

\section{Pediatric Fatty Liver Disease}

As in adults, NAFLD comprises a spectrum of diseases ranging from isolated hepatic steatosis, hepatocellular injury with necro inflammation and fibrosis (NASH) and, finally cirrhosis.

Isolated steatosis in children is similar to adult steatosis with the most common form in both being macrovesicular steatosis. Histological criteria for differentiating NAFLD from NASH in pediatric cases are the same as the criteria for adults. However, there is a lesser degree of inter-observer agreement over pediatric than adult interpretations of NAFLD [40]. This is partly because ballooning degeneration, MDB and classic zone 3 fibrosis are less common in children with definite NASH. In addition there is a separate distinctive pattern of NAFLD with fibrosis that is seen almost exclusively in children that complicates interpretation of biopsies.

An evaluation of 100 pediatric NAFLD biopsy samples [17] and subsequent cluster analysis of lesions identified two types of NAFLD patterns. Type 1, the adult NASH pattern, was more common in girls and comprised $17 \%$ of the total. Type 2 was found in $51 \%$ of the children and showed panacinar steatosis or accentuation of periportal steatosis (zone 1), zero to minimal hepatocellular ballooning with or without portal inflammation and fibrosis. Boys outnumbered girls in this category. In 16\% of patients there was an overlap of type 1 and 2 and isolated steatosis was seen in $16 \%$.

Further studies of variation of histologic patterns of NAFLD verified the general trend of a minority of children having the adult (zone 3 ) pattern of NASH with most biopsy findings placed in the overlap category (51\% and $82 \%$ respectively) than in the type 2 category [ $41-$ 42]. The differences in absolute percentages may be due to differing ethnicity of the cohorts studied i.e. Caucasians versus predominantly Asian and Hispanic).

The NASH CRN has prospectively categorized this distinctive pattern of pediatric liver disease as the "zone 1" pattern, in order to differentiate from the more classic zone 3 centered injury pattern of adult NASH. The NIDDK NASH CRN prospective study of 177 children between the ages of 6 and 17 found a predominance of adult NASH pattern (31\%) with zone 1 pattern being represented in $28 \%$ of the cases. Older children (adolescents) and boys comprised the majority of the patients (77\%) in line with previous published studies. 
Children with the zone 1 pattern tended to be younger and less insulin resistant than those with typical adult-type steatohepatitis [43].

From the above, it is evident that spectrum of NAFLD histology differs in childhood. While the classic, zone $3 \mathrm{NASH}$ pattern is characterized by the presence of steatosis with ballooning degeneration and/or perisinusoidal fibrosis in absence of portal features, the distinctive zone 1 pattern seen in children is defined by the presence of zone 1 to panacinar steatosis along with portal inflammation and/or portal fibrosis in the absence of ballooning degeneration and perisinusoidal fibrosis (Table 1).

Whether these differences in liver histopathology between pediatric and adult NAFLD are due to differences in pathogenesis or represent two phenotypes in children that ultimately evolve into a typical adult pattern of NAFLD/NASH over time remains unclear. The observation of classic NASH in older children who are probably undergoing pubertal hormonal changes adds emphasis to the proposed role of insulin resistance in NASH [44].

In summary, the zone 1 pattern and the more typical zone 3 pattern of NASH are both seen in pediatric NAFLD and are associated with different clinical, demographic and possibly, pathophysiologic features. Children with the zone 1 pattern tended to be younger, male and more obese than those with classic NASH. Children of Asian or Native American race and those of Hispanic ethnicity may be more at risk for the zone 1 pattern. These factors may have implications for understanding the genetics, natural history or response to treatment in pediatric NAFLD. It is not known whether the zone 1 pattern evolves into more typical $\mathrm{NASH}$ as the children grow older.

\section{Histologic scoring system}

Like the chronic hepatitis scoring systems, the existing NAFLD scoring systems are more useful for research than diagnosis. The first system for histologic grading of NASH was proposed based upon biopsies from adult patients [45]. This system for semi-quantitative type of evaluation was based on the idea that the histologic diagnosis of NASH relies on a constellation of features rather than on any one feature. Such an approach was recently refined in the NIDDK NASH CRN study [40] to provide a semi-quantitative feature based scoring system for NAFLD for both pediatric and adult populations. In this scoring system, histological features are grouped into five categories: steatosis, inflammation, hepatocellular injury, fibrosis and miscellaneous features. This method was derived by a group after multiple blinded readings of the study set and has been validated as such. More important, it has been demonstrated that agreement between pathologists in adult cases show reasonable concordance with the main categories of pathologic features, including steatosis, fibrosis and ballooning injury with weighted kappa values over 0.5 . Since its publication, the NASH CRN system has been used effectively to monitor histologic changes in many studies, including the NASH CRN's PIVENS trial [18].

The scoring system is composed of 14 histologic features; four of the key features are evaluated semi-quantitatively including steatosis (0-3), lobular inflammation (0-2), hepatocellular ballooning $(0-2)$ and fibrosis $(0-4)$. As with viral chronic hepatitis, fibrosis is separately assessed using a 5 stage scale ranging from no fibrosis to cirrhosis that pays particular attention to the evaluation of the intensity of perisinusoidal fibrosis. Stage 1 is subdivided into three sub-stages to differentiate between delicate perisinusoidal zone 3 fibrosis (stage 1a), dense perisinusoidal zone 3 fibrosis (stage 1b) and portal fibrosis only (stage 1c). Stage 1c is seen mostly in severely obese patients and in pediatric NASH.

Beginning with new patient enrollment into the NASH CRN, portal inflammation was also graded on a scale from 0 to 2 [26]. The other eight features: microvesicular steatosis, 
acidophil bodies, microgranulomas, lipogranulomas, pigmented macrophages, megamitochondria, Mallory hyaline and glycogenated nuclei are qualitatively assessed as present or absent. The entire system is used to assess histology in patient biopsies in the NASH CRN, but disease activity is assessed as a sum of three components into a NAFLD Activity Score (NAS).

The NAS is defined as the unweighted sum of the scores for steatosis (0-3), lobular inflammation (0-3) and ballooning (0-2). Although the authors emphasized that the score should not be used to derive the diagnosis of NASH, because of its design, higher scores do correlate with a higher frequency of diagnosing NASH. Unfortunately some publications have considered the NAS of $\geq 5$ to be synonymous with the diagnosis of NASH. A recent study by the NASH CRN evaluated the NAS against the histopathological diagnosis of NASH and found that not all biopsies with NAS of $\geq 5$ have findings that meet diagnostic criteria of steatohepatitis and that many cases where NAS $\leq 4$ did. The authors reemphasized that, although a diagnosis of definite NASH is more likely to be made at higher scores, the score should not used to decide whether or not a biopsy shows NASH. In addition, they advise that the exercise of diagnosis and scoring while leading to inter-related results, serve distinct, separate and important purposes [46].

One important parameter in the scoring of liver biopsies for diagnostic and research purposes is the length of the biopsy which needs to be at least $16 \mathrm{~mm}$ [47] and have a diameter of 1.2 to $1.8 \mathrm{~mm}$. This can be estimated based on the presence of at least 10 portal tracts of reasonable size which are not transected by a very narrow needle precluding their near complete visualization.

In conclusion, the diagnosis of NAFLD relies on the patterns of lesions within the hepatic parenchyma and a simple listing or scoring of lesions is not considered adequate for diagnosis [46]. In other words, NAS is not intended to be used as a diagnostic tool, but rather to provide a uniform tool for assessing disease severity and ideally in clinical trials such as natural history studies and studies assessing treatment response.

\section{Summary}

The worldwide rise of obesity is reflected in the increased prevalence of NAFLD making more likely for a pathologist to see these changes in liver biopsies. The histological pattern of NAFLD in adults is distinct; however, children with NAFLD have a significant range of histological patterns which makes the diagnosis more challenging. Similarly, the morphologic pathogenesis of NAFLD is better understood in adults than in children. Specifically, there are no natural history studies showing the evolution of the zone 1 pattern in children, whether it evolves to the adult pattern with increasing age and what processes are involved in progression to cirrhosis. The NAS scoring system is the cornerstone of studies in NAFLD but cannot be used in diagnosis of NAFLD. The ongoing NASH CRN study is expected to continue to yield significant histologic, genetic, biochemical and radiological parameters involved in development, progression, regression/resolution and treatment of NAFLD in adults and children.

\section{Acknowledgments}

This work was supported by the Intramural Research Program of the NIH, National Cancer Institute.

\section{REFERENCES}

1. Hossain P, Kawar B, El Nahas M. Obesity and diabetes in the developing world--a growing challenge. N Engl J Med. 2007; 356:213-215. [PubMed: 17229948] 
2. Subramanian V, Ferrante AW Jr. Obesity, inflammation, and macrophages. Nestle Nutr Workshop Ser Pediatr Program. 2009; 63:151-159. discussion 159-62, 259-68.

3. Third Report of the National Cholesterol Education Program (NCEP) Expert Panel on Detection, Evaluation, and Treatment of High Blood Cholesterol in Adults (Adult Treatment Panel III) final report. Circulation. 2002; 106:3143-3421. [PubMed: 12485966]

4. Rafiq N, Bai C, Fang Y, et al. Long-term follow-up of patients with nonalcoholic fatty liver. Clin Gastroenterol Hepatol. 2009; 7:234-238. [PubMed: 19049831]

5. Marchesini G, Brizi M, Bianchi G, et al. Nonalcoholic fatty liver disease: a feature of the metabolic syndrome. Diabetes. 2001; 50:1844-1850. [PubMed: 11473047]

6. Silverman JF, O'Brien KF, Long S, et al. Liver pathology in morbidly obese patients with and without diabetes. Am J Gastroenterol. 1990; 85:1349-1355. [PubMed: 2220728]

7. Wanless IR, Lentz JS. Fatty liver hepatitis (steatohepatitis) and obesity: an autopsy study with analysis of risk factors. Hepatology. 1990; 12:1106-1110. [PubMed: 2227807]

8. Alberti KG, Zimmet P, Shaw J. The metabolic syndrome--a new worldwide definition. Lancet. 2005; 366:1059-1062. [PubMed: 16182882]

9. Neuschwander-Tetri BA, Caldwell SH. Nonalcoholic steatohepatitis: summary of an AASLD Single Topic Conference. Hepatology. 2003; 37:1202-1219. [PubMed: 12717402]

10. Einhorn D, Reaven GM, Cobin RH, et al. American College of Endocrinology position statement on the insulin resistance syndrome. Endocr Pract. 2003; 9:237-252. [PubMed: 12924350]

11. Ekstedt M, Franzen LE, Mathiesen UL, et al. Long-term follow-up of patients with NAFLD and elevated liver enzymes. Hepatology. 2006; 44:865-873. [PubMed: 17006923]

12. Fraser A, Longnecker MP, Lawlor DA. Prevalence of elevated alanine aminotransferase among US adolescents and associated factors: NHANES 1999-2004. Gastroenterology. 2007; 133:18141820. [PubMed: 18054554]

13. Genuth S, Alberti KG, Bennett P, et al. Follow-up report on the diagnosis of diabetes mellitus. Diabetes Care. 2003; 26:3160-3167. [PubMed: 14578255]

14. Schwimmer JB, Deutsch R, Kahen T, et al. Prevalence of fatty liver in children and adolescents. Pediatrics. 2006; 118:1388-1393. [PubMed: 17015527]

15. Strauss RS, Barlow SE, Dietz WH. Prevalence of abnormal serum aminotransferase values in overweight and obese adolescents. J Pediatr. 2000; 136:727-733. [PubMed: 10839867]

16. Manco M, Bedogni G, Marcellini M, et al. Waist circumference correlates with liver fibrosis in children with non-alcoholic steatohepatitis. Gut. 2008; 57:1283-1287. [PubMed: 18218674]

17. Schwimmer JB, Behling C, Newbury R, et al. Histopathology of pediatric nonalcoholic fatty liver disease. Hepatology. 2005; 42:641-649. [PubMed: 16116629]

18. Sanyal AJ, Chalasani N, Kowdley KV, et al. Pioglitazone, vitamin E, or placebo for nonalcoholic steatohepatitis. N Engl J Med. 362:1675-1685. [PubMed: 20427778]

19. Tandra SY. MM Presence and Significance of Microvesicular Steatosis in Nonalcoholic Fatty Liver Disease. Journal of Hepatology. 2010

20. Chalasani N, Wilson L, Kleiner DE, et al. Relationship of steatosis grade and zonal location to histological features of steatohepatitis in adult patients with non-alcoholic fatty liver disease. $\mathrm{J}$ Hepatol. 2008; 48:829-834. [PubMed: 18321606]

21. Lackner C, Gogg-Kamerer M, Zatloukal K, et al. Ballooned hepatocytes in steatohepatitis: the value of keratin immunohistochemistry for diagnosis. J Hepatol. 2008; 48:821-828. [PubMed: 18329127]

22. Straub BK, Stoeffel P, Heid H, et al. Differential pattern of lipid droplet-associated proteins and de novo perilipin expression in hepatocyte steatogenesis. Hepatology. 2008; 47:1936-1946. [PubMed: 18393390]

23. Fujii H, Ikura Y, Arimoto J, et al. Expression of perilipin and adipophilin in nonalcoholic fatty liver disease; relevance to oxidative injury and hepatocyte ballooning. J Atheroscler Thromb. 2009; 16:893-901. [PubMed: 20032580]

24. Caldwell SH, Chang CY, Nakamoto RK, et al. Mitochondria in nonalcoholic fatty liver disease. Clin Liver Dis. 2004; 8:595-617. x. [PubMed: 15331066] 
25. Lefkowitch JH, Haythe JH, Regent N. Kupffer cell aggregation and perivenular distribution in steatohepatitis. Mod Pathol. 2002; 15:699-704. [PubMed: 12118106]

26. Brunt EM, Kleiner DE, Wilson LA, et al. Portal chronic inflammation in nonalcoholic fatty liver disease (NAFLD): a histologic marker of advanced NAFLD-Clinicopathologic correlations from the nonalcoholic steatohepatitis clinical research network. Hepatology. 2009; 49:809-820. [PubMed: 19142989]

27. Brunt EM, Ramrakhiani S, Cordes BG, et al. Concurrence of histologic features of steatohepatitis with other forms of chronic liver disease. Mod Pathol. 2003; 16:49-56. [PubMed: 12527713]

28. Sanyal AJ, Contos MJ, Sterling RK, et al. Nonalcoholic fatty liver disease in patients with hepatitis $\mathrm{C}$ is associated with features of the metabolic syndrome. Am J Gastroenterol. 2003; 98:20642071. [PubMed: 14499789]

29. Nakayama H, Itoh H, Kunita S, et al. Presence of perivenular elastic fibers in nonalcoholic steatohepatitis Fibrosis Stage III. Histol Histopathol. 2008; 23:407-409. [PubMed: 18228197]

30. Harrison SA, Brunt EM, Goodman ZD, et al. Diabetic hepatosclerosis: diabetic microangiopathy of the liver. Arch Pathol Lab Med. 2006; 130:27-32. [PubMed: 16390234]

31. Syn WK, Jung Y, Omenetti A, et al. Hedgehog-mediated epithelial-to-mesenchymal transition and fibrogenic repair in nonalcoholic fatty liver disease. Gastroenterology. 2009; 137:1478-1488. e8. [PubMed: 19577569]

32. Richardson MM, Jonsson JR, Powell EE, et al. Progressive fibrosis in nonalcoholic steatohepatitis: association with altered regeneration and a ductular reaction. Gastroenterology. 2007; 133:80-90. [PubMed: 17631134]

33. Mendler MH, Turlin B, Moirand R, et al. Insulin resistance-associated hepatic iron overload. Gastroenterology. 1999; 117:1155-1163. [PubMed: 10535879]

34. Vigano M, Vergani A, Trombini P, et al. Insulin resistance influence iron metabolism and hepatic steatosis in type II diabetes. Gastroenterology. 2000; 118:986-987. [PubMed: 10841644]

35. George DK, Goldwurm S, MacDonald GA, et al. Increased hepatic iron concentration in nonalcoholic steatohepatitis is associated with increased fibrosis. Gastroenterology. 1998; 114:311-318. [PubMed: 9453491]

36. Woods JR Jr, Plessinger MA, Fantel A. An introduction to reactive oxygen species and their possible roles in substance abuse. Obstet Gynecol Clin North Am. 1998; 25:219-236. [PubMed: 9547768]

37. Chitturi S, Weltman M, Farrell GC, et al. HFE mutations, hepatic iron, and fibrosis: ethnic-specific association of NASH with C282Y but not with fibrotic severity. Hepatology. 2002; 36:142-149. [PubMed: 12085358]

38. Younossi ZM, Gramlich T, Bacon BR, et al. Hepatic iron and nonalcoholic fatty liver disease. Hepatology. 1999; 30:847-850. [PubMed: 10498632]

39. Nelson JE, Wilson L, Brunt EM, et al. Relationship between the pattern of hepatic iron deposition and histological severity in nonalcoholic fatty liver disease. Hepatology. 53:448-457. [PubMed: 21274866]

40. Kleiner DE, Brunt EM, Van Natta M, et al. Design and validation of a histological scoring system for nonalcoholic fatty liver disease. Hepatology. 2005; 41:1313-1321. [PubMed: 15915461]

41. Nobili V, Marcellini M, Devito R, et al. NAFLD in children: a prospective clinical-pathological study and effect of lifestyle advice. Hepatology. 2006; 44:458-465. [PubMed: 16871574]

42. Carter-Kent C, Yerian LM, Brunt EM, et al. Nonalcoholic steatohepatitis in children: a multicenter clinicopathological study. Hepatology. 2009; 50:1113-1120. [PubMed: 19637190]

43. Patton HM, Lavine JE, Van Natta ML, et al. Clinical correlates of histopathology in pediatric nonalcoholic steatohepatitis. Gastroenterology. 2008; 135:1961-1971. e2. [PubMed: 19013463]

44. Marchesini G, Brizi M, Morselli-Labate AM, et al. Association of nonalcoholic fatty liver disease with insulin resistance. Am J Med. 1999; 107:450-455. [PubMed: 10569299]

45. Brunt EM, Janney CG, Di Bisceglie AM, et al. Nonalcoholic steatohepatitis: a proposal for grading and staging the histological lesions. Am J Gastroenterol. 1999; 94:2467-2474. [PubMed: 10484010] 
46. Brunt EM, Kleiner DE, Wilson LA, et al. Nonalcoholic fatty liver disease (NAFLD) activity score and the histopathologic diagnosis in NAFLD: distinct clinicopathologic meanings. Hepatology. 2011; 53:810-820. [PubMed: 21319198]

47. Goldstein NS, Hastah F, Galan MV, et al. Fibrosis heterogeneity in nonalcoholic steatohepatitis and hepatitis C virus needle core biopsy specimens. Am J Clin Pathol. 2005; 123:382-387.

[PubMed: 15716234] 


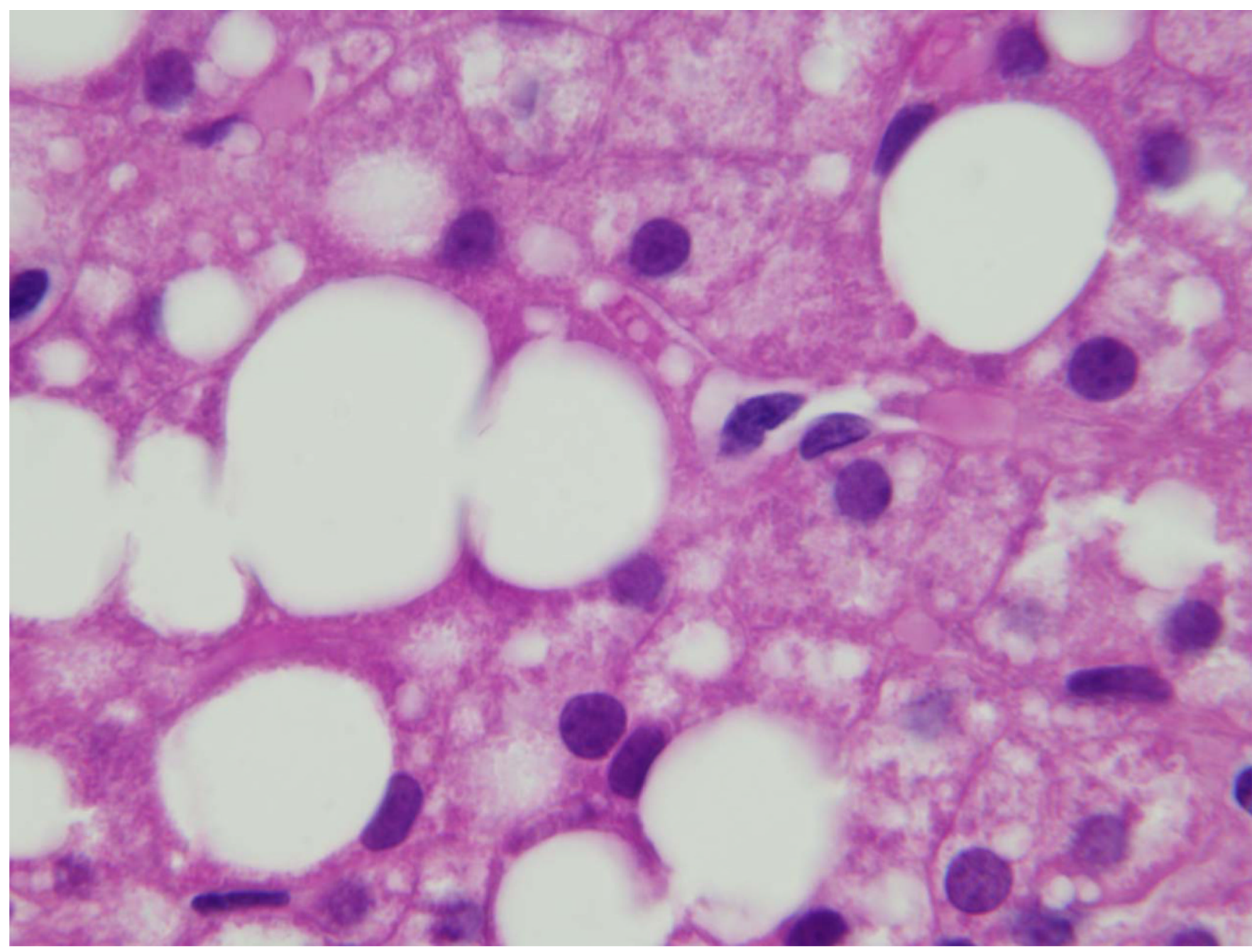

Figure 1.

Hepatocytes with macrovesicular steatosis appearing as a large lipid droplet and smaller droplets. 


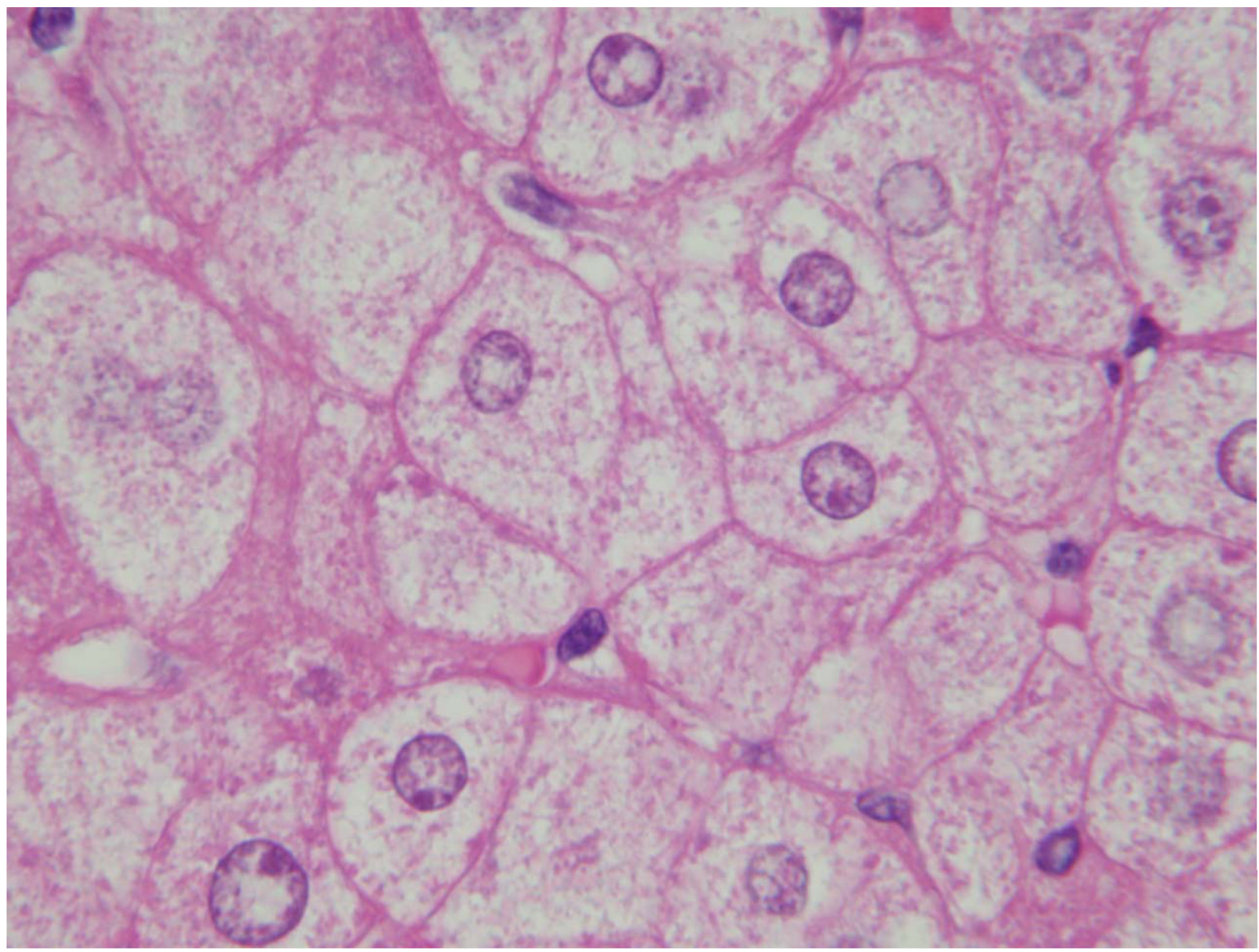

Figure 2.

Foamy appearance of the cytoplasm in contiguous hepatocytes depicting microvesicular steatosis. 


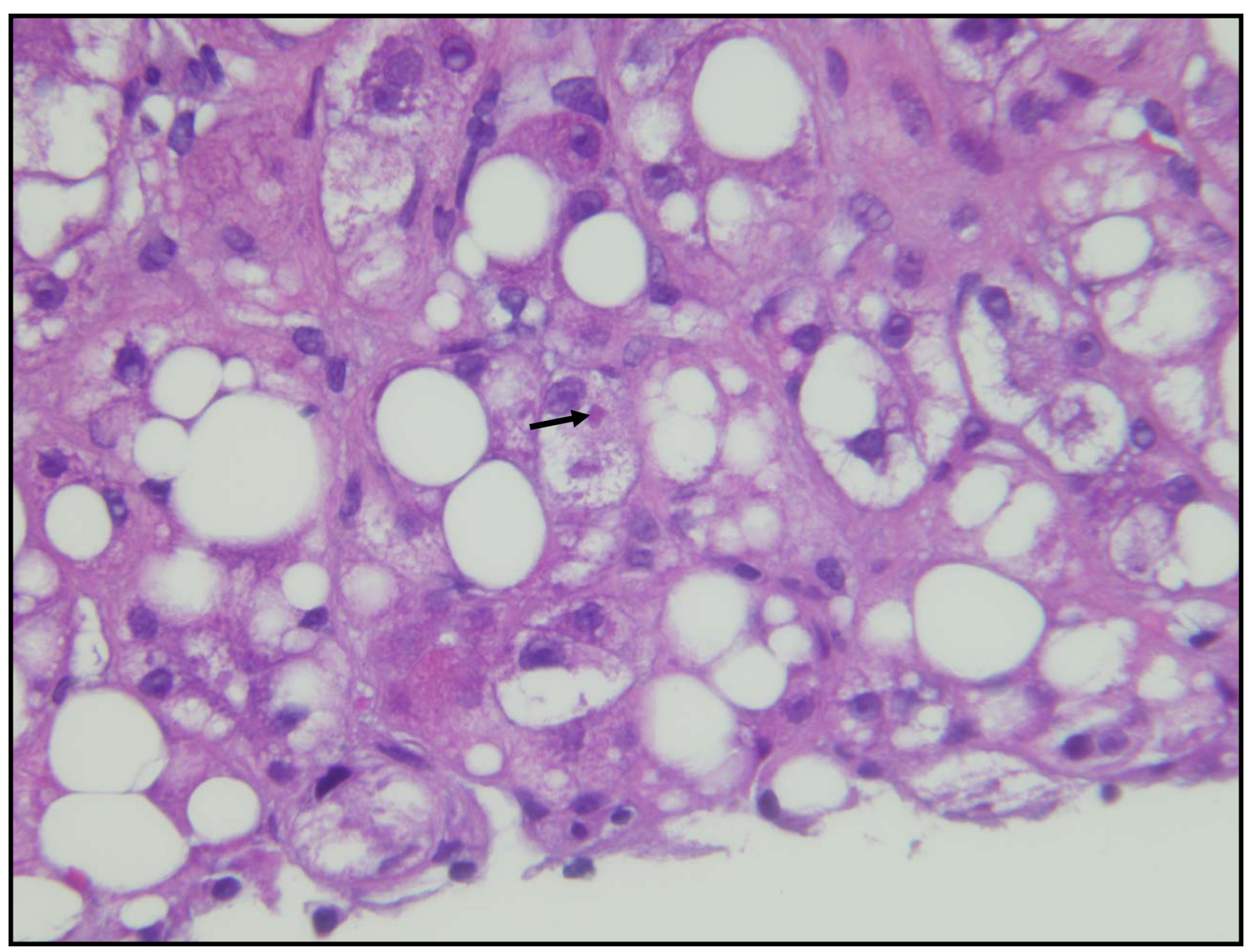

Figure 3.

Megamitochondria appearing as refractile hyaline material within hepatocytes. 


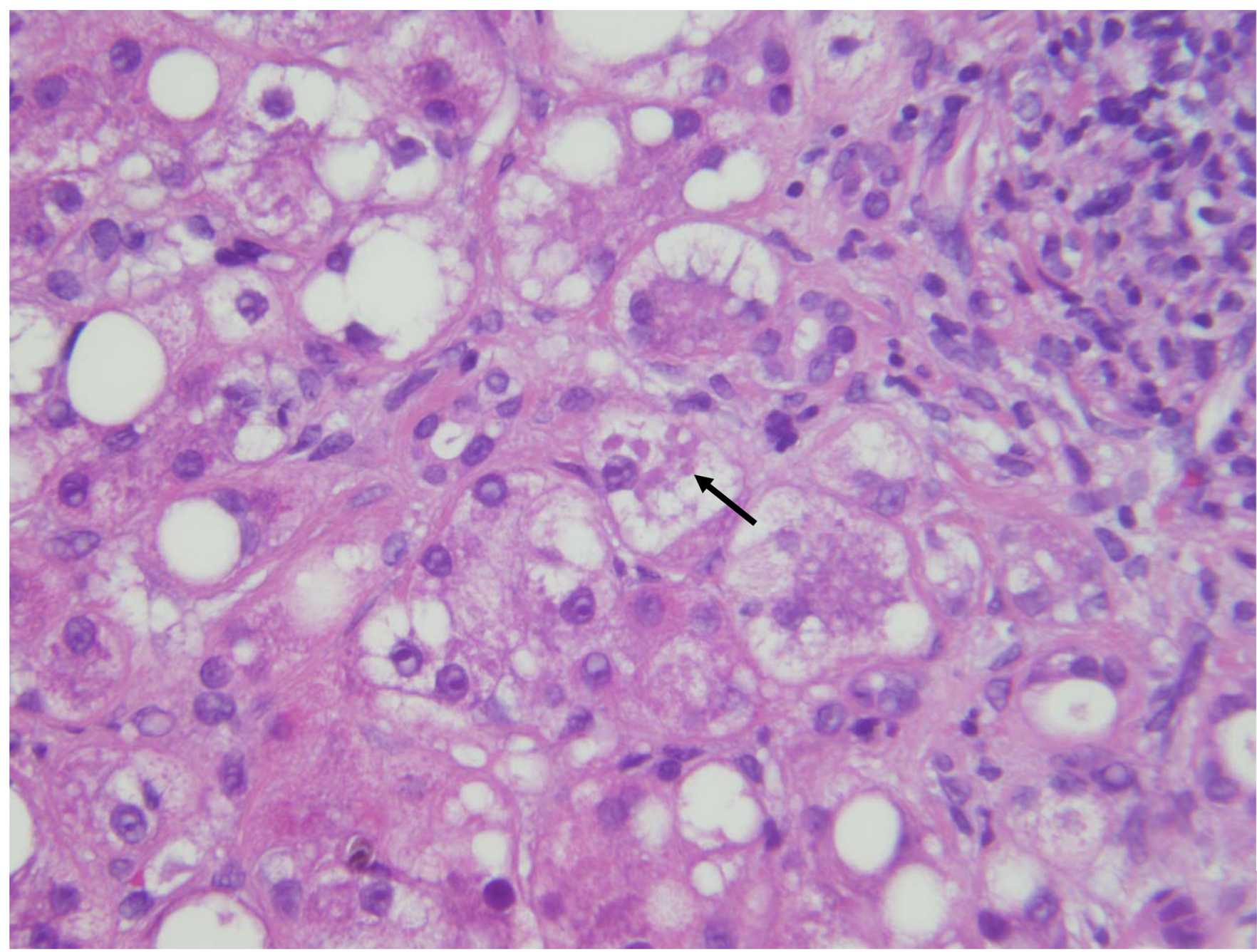

Figure 4.

Ballooning cell changes and Mallory Denk Bodies in some of the ballooned hepatocytes. 


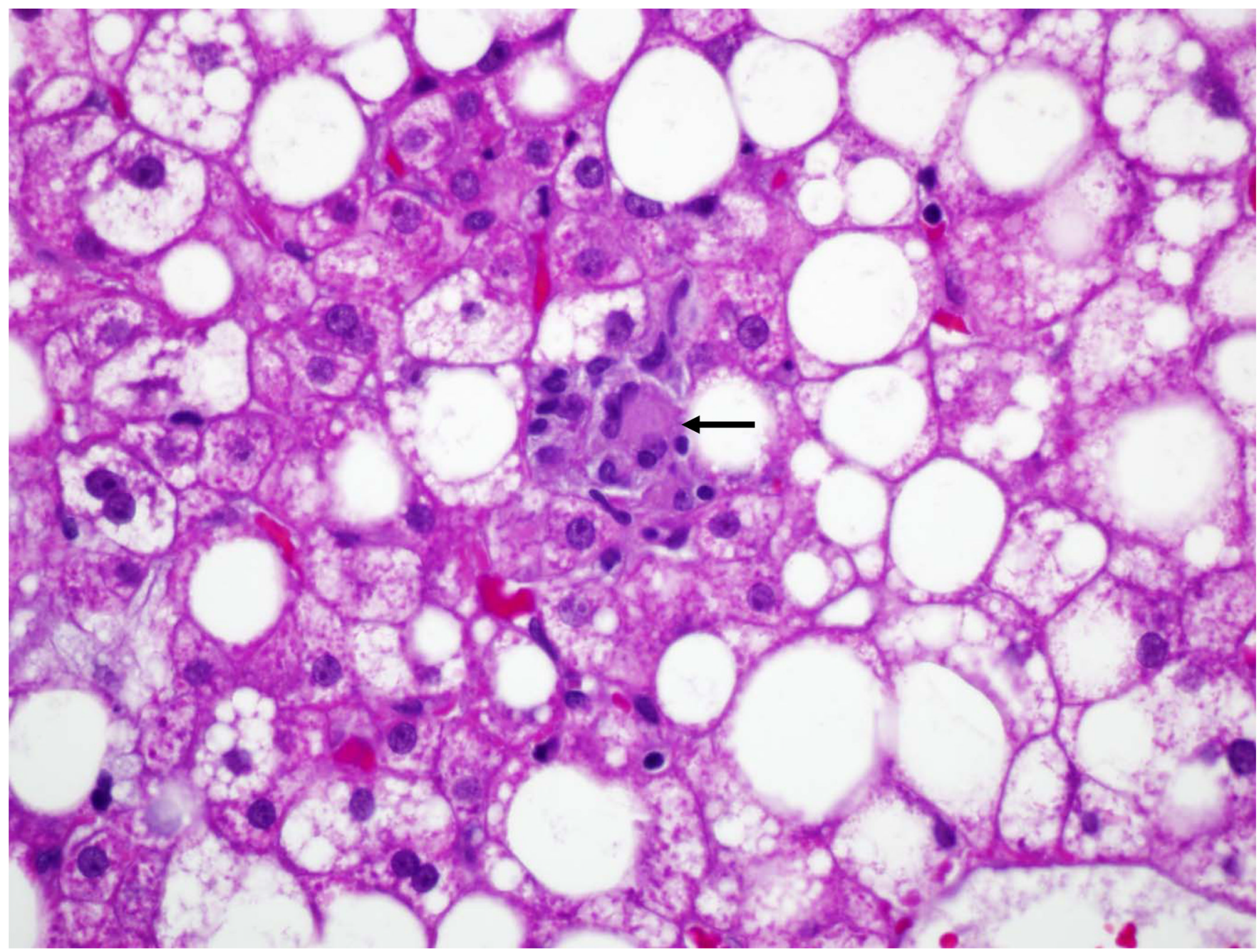

Figure 5.

Kuppfer cells with ingested lipid droplets (microgranulomas). 


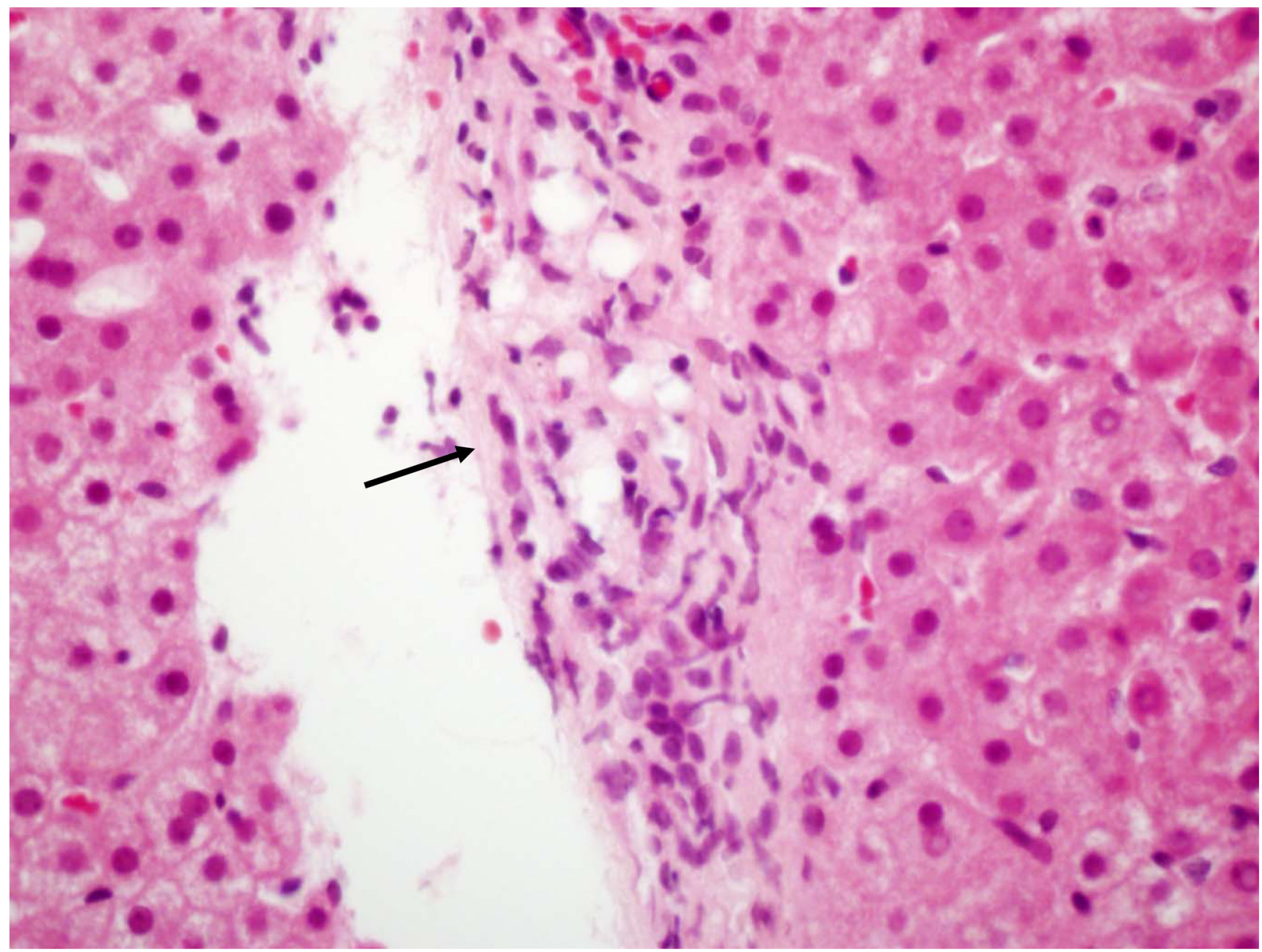

Figure 6.

Macrophages containing lipid droplets (lipogranuloma) with associated local fibrosis. 


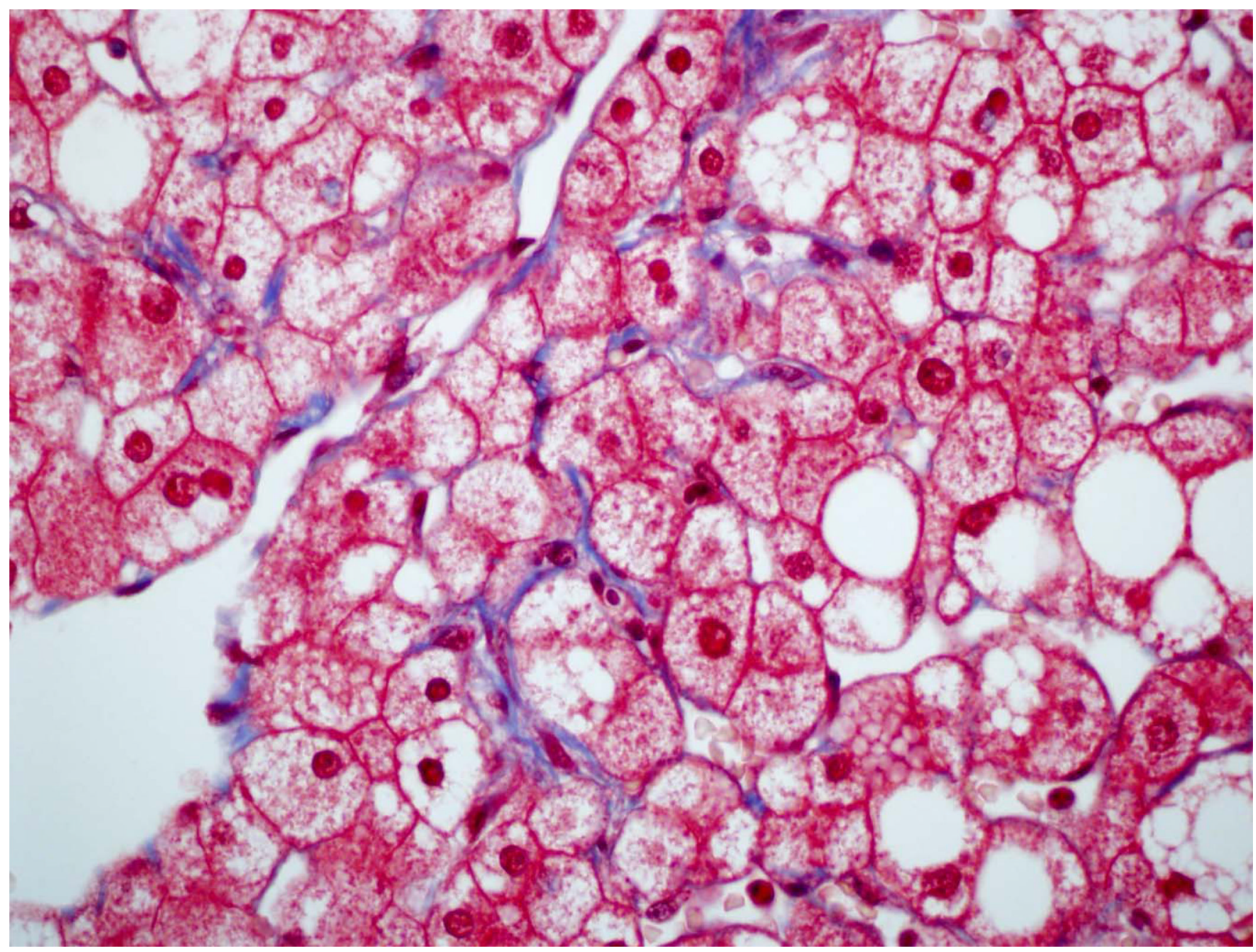

Figure 7.

Delicate perisinusoidal fibrosis (Stage 1a) demonstrated on Masson trichrome. 
Table 1

Comparison of the two distinct NAFLD patterns

\begin{tabular}{|l|l|l|}
\hline & Zone 3 Pattern & Zone 1 Pattern \\
\hline Steatosis & Zone 3 to Panacinar & Zone 1 to Panacinar \\
\hline Ballooning & Present & Usually Absent \\
\hline Mallory-Denk bodies & May be present & Usually Absent \\
\hline Fibrosis & Zone 3 perisinusoidal & Periportal then portal-portal BF \\
\hline
\end{tabular}

